\title{
Insights into the use of thermography to assess burn wound healing potential: a reliable and valid technique when compared to laser Doppler imaging
}

Mariëlle E. H. Jaspers

Ilse Maltha

John H. G. M. Klaessens

Henrica C. W. de Vet

Rudolf M. Verdaasdonk

Paul P. M. van Zuijlen 


\title{
Insights into the use of thermography to assess burn wound healing potential: a reliable and valid technique when compared to laser Doppler imaging
}

\author{
Mariëlle E. H. Jaspers, ${ }^{a, b, c, d, e, *}$ Ilse Maltha, ${ }^{a}$ John H. G. M. Klaessens, ${ }^{f}$ Henrica C. W. de Vet, ${ }^{g, h}$ \\ Rudolf M. Verdaasdonk, ${ }^{f}$ and Paul P. M. van Zuijlen ${ }^{a, b, c, d, e}$ \\ aBurn Center, Red Cross Hospital, P.O. Box 1074, 1940 EB Beverwijk, The Netherlands \\ ${ }^{b}$ Association of Dutch Burn Centers, P.O. Box 1015, 1940 EA Beverwijk, The Netherlands \\ ${ }^{c}$ Red Cross Hospital, Department of Plastic, Reconstructive and Hand Surgery, P.O. Box 1074, 1940 EB Beverwijk, The Netherlands \\ ${ }^{d}$ VU Medical Center, Department of Plastic, Reconstructive and Hand Surgery, P.O. Box 7057, 1007 MB Amsterdam, The Netherlands \\ ${ }^{e}$ Research Institute MOVE VU University Medical Center of Amsterdam, P.O. Box 7057, 1007 MB Amsterdam, The Netherlands \\ fVU University Medical Center, Department of Physics and Medical Technology, P.O. Box 7057, 1007 MB Amsterdam, The Netherlands \\ gVU University Medical Center, Department of Epidemiology and Biostatistics, P.O. Box 7057, 1007 MB Amsterdam, The Netherlands \\ hVU University Medical Center, EMGO Institute for Health and Care Research, P.O. Box 7057, 1007 MB Amsterdam, The Netherlands
}

\begin{abstract}
Adequate assessment of burn wounds is crucial in the management of burn patients. Thermography, as a noninvasive measurement tool, can be utilized to detect the remaining perfusion over large burn wound areas by measuring temperature, thereby reflecting the healing potential (HP) (i.e., number of days that burns require to heal). The objective of this study was to evaluate the clinimetric properties (i.e., reliability and validity) of thermography for measuring burn wound HP. To evaluate reliability, two independent observers performed a thermography measurement of 50 burns. The intraclass correlation coefficient (ICC), the standard error of measurement (SEM), and the limits of agreement (LoA) were calculated. To assess validity, temperature differences between burned and nonburned skin $(\Delta T)$ were compared to the HP found by laser Doppler imaging (serving as the reference standard). By applying a visual method, one $\Delta T$ cutoff point was identified to differentiate between burns requiring conservative versus surgical treatment. The ICC was 0.99 , expressing an excellent correlation between two measurements. The SEM was calculated at $0.22^{\circ} \mathrm{C}$, the LoA at $-0.58^{\circ} \mathrm{C}$ and $0.64^{\circ} \mathrm{C}$. The $\Delta T$ cutoff point was $-0.07^{\circ} \mathrm{C}$ (sensitivity $80 \%$; specificity $80 \%$ ). These results show that thermography is a reliable and valid technique in the assessment of burn wound HP. ๑ 2016 Society of Photo-Optical Instrumentation Engineers (SPIE) [DOI: 10 .1117/1.JBO.21.9.096006]
\end{abstract}

Keywords: thermography; laser Doppler imaging; burns; burn wound healing potential; reliability; validity.

Paper 160334RR received May 25, 2016; accepted for publication Aug. 24, 2016; published online Sep. 13, 2016.

\section{Introduction}

Adequate assessment of burn wound healing potential (HP) is crucial in the management of burn patients. Clinical (subjective) evaluation is the most widely used method for determining the expected burn wound outcome. This type of assessment is based on the probability of whether a wound will heal spontaneously ( $<3$ weeks) or requires surgical therapy. This distinction in healing time is made, as wounds with a low HP $(>3$ weeks) are correlated with a significantly lower scar quality. ${ }^{1,2}$ Thus, underestimation of the healing time may lead to an increased risk of pathological scar formation, whereas overestimation of the healing time may increase the amount of needless surgery. It is easy to identify the mild injury of sunburn or to discern the other extreme: a dry, inelastic, insensitive, cadaveric-appearing wound that reflects serious injury to the skin. However, when a burn wound is first evaluated it is often difficult to determine the subtle differences and its potential to heal. Accordingly, clinical evaluation is not always sufficient as it is accurate in only $70 \%$ of the cases. ${ }^{3}$ This accuracy is even lower for inexperienced surgeons,

*Address all correspondence to: Mariëlle E. H. Jaspers, E-mail: mjaspers@ @rkz . $\mathrm{nl}$ around 50\%. ${ }^{4,5}$ Therefore, objective tools that improve the assessment of burn wound HP are of great relevance.

Currently, laser Doppler imaging (LDI) is the most widely used noninvasive measurement tool for the assessment of burn wounds and the only technique that has been approved by the U.S. Food and Drug Administration. The working mechanism of LDI is based on the Doppler principle. Laser light that is directed at moving erythrocytes in sampled tissue exhibits a frequency change that is proportional to the amount of perfusion in the tissue. A lower perfusion correlates with a lower HP and thus a more severe burn wound. ${ }^{6}$ LDI is a valid measurement tool, providing $>95 \%$ accuracy (compared to histology, clinical assessment, and/or outcome) in measuring burn wound HP, if scanning is performed between $48 \mathrm{~h}$ and 5 days postburn. ${ }^{7-9}$ However, the use of LDI is accompanied by some disadvantages. The current commercial device available for clinical use is rather costly and cumbersome. Positioning, scanning, and evaluating an area of $50 \times 50 \mathrm{~cm}^{2}$ can take several minutes. Furthermore, it is important that the patient remain still during imaging, since any movement will result in scanning artifacts. This can be a challenging process, especially in children.

$1083-3668 / 2016 / \$ 25.00$ @ 2016 SPIE 
Thermography, or thermal imaging, is a noninvasive measurement technique based on the burn wound temperature as an indicator of its prognosis. ${ }^{10}$ Due to the fact that the vascular perfusion is destroyed in severe burn wounds, they tend to be colder than healthy skin. Adversatively, in less severe burns with an expected healing time $<14$ days, the perfusion is mainly intact. Due to loss of the epidermal layer in these burns, the existing hyperemia is measurable at the surface. As a result, a higher temperature than healthy skin will be assessed. These hypotheses were described by Hackett, ${ }^{11}$ who performed one of the largest studies on thermography in burn patients. Over the years, thermal cameras have evolved and refined, allowing real-time infrared imaging and detection of temperature differences as small as $0.05^{\circ} \mathrm{C}$. Thermal images of large areas can be captured within seconds. In addition, the cameras have recently become less expensive $(<\$ 800)$ and are small and easy to use. These characteristics make thermography applicable in routine clinical practice. Accordingly, the technique has regained attention with promising results. ${ }^{12-14}$ However, before implementing a measurement tool in clinical practice, it is essential to test its clinimetric properties (i.e., reliability and validity). ${ }^{15,16}$ Until now, no clinimetric evaluation has been performed on thermography in burns. Therefore, the objective of this study was to assess the reliability and validity of thermography for measuring burn wound HP.

\section{Materials and Methods}

\subsection{Study Population}

Patients, age $\geq 18$ years, with acute burn wounds were included from July 2014 to May 2015. Unconscious patients [due to a large total body surface area (TBSA) burned] were not included as they were not able to give informed consent. In addition, we did not include patients with a suspected wound infection. The required sample size in this clinimetric study was estimated at 50 burn wounds, based on a $95 \%$ confidence interval (CI) of $0.1 .^{15}$ Measurements were performed in the Red Cross Hospital in Beverwijk, The Netherlands, either at the outpatient clinic or during admission at the Burn Center. The regional Medical Ethics Committee approved the study protocol (reference No. M014-002) and agreed that this study did not fall under the scope of the Medical Research involving Human Subjects Act because patients were not subjected to specific actions, and/or were not dictated to activities as stated in the Medical Research involving Human Subjects Act. However, according to the Declaration of Helsinki, written informed consent was obtained from all patients.

\subsection{Reference Standard/Laser Doppler Imaging}

Of every burn wound, one LDI measurement was acquired to obtain a reference value. LDI measurements were performed using the moorLDI2-Burn Imager $^{\mathrm{TM}}$ (Moor Instruments, Axminster, United Kingdom) with a wavelength of $785 \mathrm{~nm}$. The moorLDI-Burn software version V3.0 was used for the analysis. The moorLDI2 Imager contains a CCD camera with $2592 \times 1944$ pixel resolution. The spatial resolution is up to $256 \times 256$ pixels: $0.2 \mathrm{~mm} /$ pixel at $20 \mathrm{~cm}$ and $2 \mathrm{~mm} /$ pixel at $100 \mathrm{~cm}$ (camera distance to the scanned area). The bandwidth was $250 \mathrm{~Hz}$ to $15 \mathrm{kHz}$. Measurements were obtained between $48 \mathrm{~h}$ and 5 days postburn according to the guidelines. LDI is based on the principle that moving red blood cells cause a

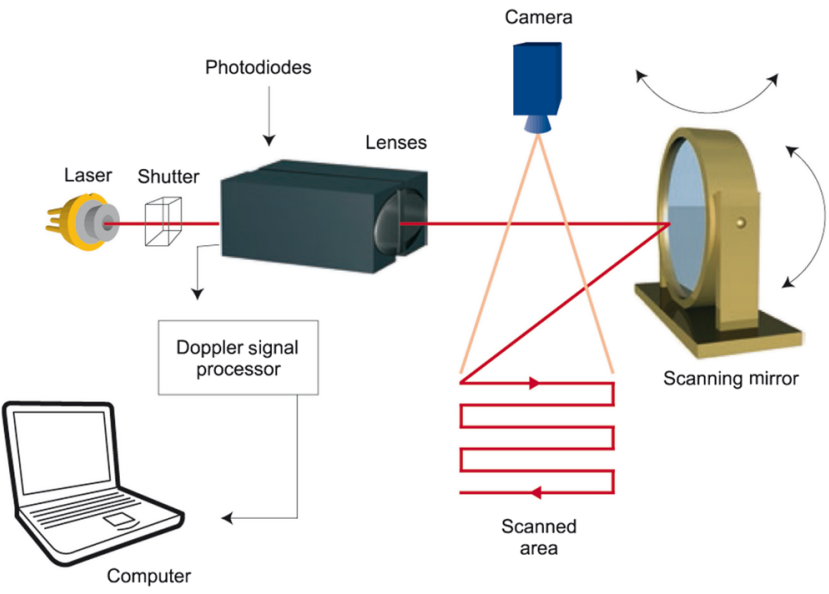

Fig. 1 Schematic overview of the moorLDI2-Burn system. Single point imaging scans a laser beam back and forth across the tissue. Laser light penetrates the skin and is scattered by moving blood cells that cause Doppler frequency shifts, which are processed to produce a color-coded blood flow map. The scan speed is $4 \mathrm{~ms} /$ pixel. An inbuilt CCD camera records a clinical color photograph at the same time to aid visualization of the scanned area. Source: from moorLDI2-BI user manual.

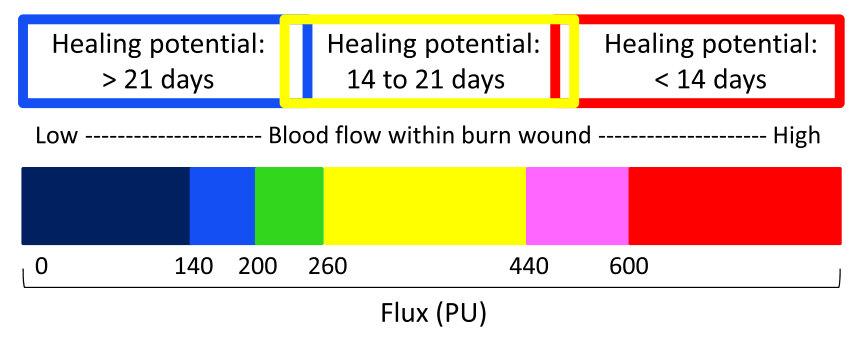

Fig. 2 LDI color codes reflecting different burn wound HPs. The color codes are based on flux (i.e., perfusion) values, expressed in perfusion units (PU). Blue: HP >21 days; 0 to 200 PU. Yellow: HP 14 to 21 days; 260 to 440 PU. Red: HP <14 days; >600 PU. Source: adapted from moorLDI2-BI user manual.

Doppler frequency shift of the laser light (Fig. 1), which is photodetected and processed to generate a line by line colorcoded map. These maps are color-coded using red, yellow, and blue related to the "flux" range (i.e., perfusion), corresponding to the HP of a burn wound $(<14,14$ to 21 , or $>21$ days $)$ (Fig. 2). ${ }^{17}$ In burn medicine, these are the accepted cutoff days because they are important for clinical decision making, and for predicting the risk of scar formation. In addition to the three principle colors, a certain amount of green and pink may also be present on the LDI scan, but in this study, we only assigned a measurement area to a specific healing category if $>75 \%$ of the flux value consisted of red, yellow, or blue. A thorough explanation on the validation of the color codes is described elsewhere. ${ }^{17,18}$

\subsection{Thermography System}

In order to obtain thermal images, the Xenics Gobi-384 (Xenics NV, Leuven, Belgium) was used. This is a compact plug-andplay infrared camera system with a spectral bandwidth of 8 to $14 \mu \mathrm{m}$. The camera contains an on board Digital Signal Processor, allowing for real-time image analysis (Xeneth 


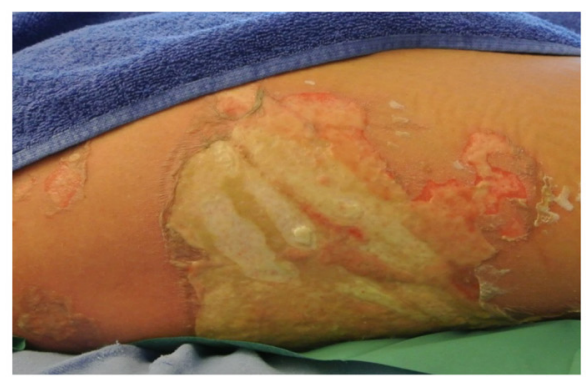

(a)

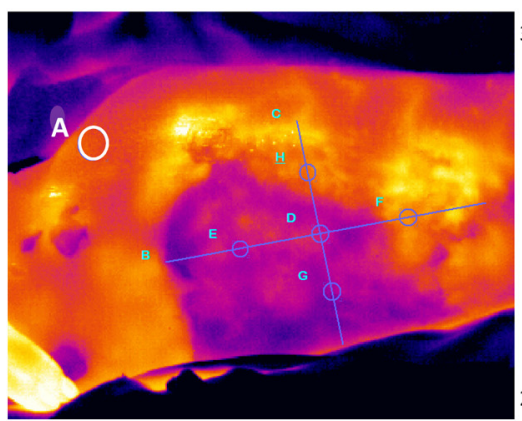

(b)

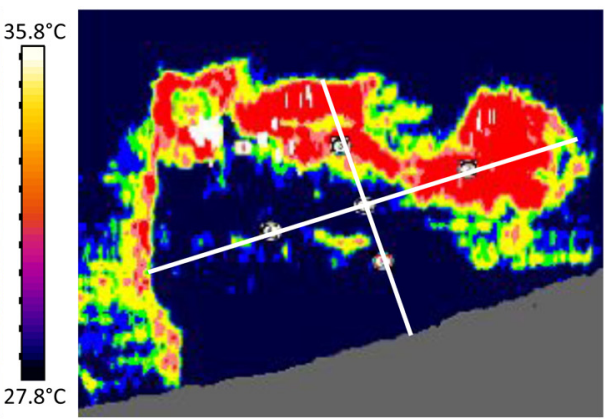

(c)

Fig. 3 Illustration of the study procedure to assess validity. (a) Normal photograph of a heterogeneous burn wound: HP $>21$ days in the center and HP $<14$ days around. (b) Thermography image with the standardized algorithm containing five measurement areas, and the reference area consisting of nonburned skin located $\pm 5 \mathrm{~cm}$ proximally to the burn wound, indicated by the letter $A$. The displayed colors concern the "iron palette." (c) LDI scan covering the three colors expressing different HPs, accompanied by the standardized algorithm.

software, Xenics NV, Leuven). The resolution of the system is $384 \times 288$ pixels with maximum frame rates of $84 \mathrm{~Hz}$. The maximum imaging time of each burn wound was $60 \mathrm{~s}$. For the analysis, we took one frame from each video clip at $15 \mathrm{f} / \mathrm{s}$. Since we performed static thermography measurements, no temperature alterations within one video clip were observed. The device is able to detect temperature differences as small as $0.05^{\circ} \mathrm{C}$. No direct contact with the skin is required. Thermography measurements were performed subsequent to the LDI measurement, after the burn wound had been cleaned with warm water and residual topical ointment was removed. To minimize the effect of warm water on the skin temperature, we allowed patients to acclimatize for $10 \mathrm{~min}$ to stable room temperature $\left(23^{\circ} \mathrm{C}\right)$. We assured that the wounds were dry to prevent lower temperature measurements due to evaporative heat loss. ${ }^{19}$ In addition, heat lamps that normally prevent warmth loss during the bandage change were turned off for the purpose of this study. Also, the ambient temperature was kept stable by the continuous air flow and climate control that is provided at the Burn Center. All thermography results were expressed as $\Delta T\left({ }^{\circ} \mathrm{C}\right)$, indicating the temperature difference between burned and nonburned skin. The nonburned site was located $\pm 5 \mathrm{~cm}$ proximally to the burn wound [Fig. 3(b)]. The displayed thermography colors concern the "iron palette."

\subsection{Study Procedure}

\subsubsection{Reliability}

In order to assess the interobserver reliability, two independent observers obtained a thermography measurement (i.e., temperature video) of each burn wound. Subsequently, the thermography videos were analyzed crosswise: both observers performed a temperature analysis of the video obtained by the other observer. This procedure was preferred because someone else than the person obtaining the video may perform the temperature analysis in clinical practice. Both observers assessed a homogeneous area within each burn wound, which was indicated on a normal photograph. To determine the reliability, we used $\Delta T$ of both analyses.

\subsubsection{Validity}

The validity was assessed by comparing the thermography results with the LDI results (Fig. 3). Within one frame of the thermography video and on the LDI color-coded map, measurement areas $(\sim 1 \mathrm{~cm})$ were selected following a standardized algorithm as described by Verhaegen et al. ${ }^{20}$ In this way, selection bias of the measurement areas was prevented. Moreover, if we selected burn wounds as a whole, temperature differences would have been leveled out because of the heterogeneous aspect (i.e., different HPs) of the wounds. Anatomical landmarks were taken into account to retrieve exactly the same measurement areas in the LDI and thermography image. In certain burn wounds, the number of measurement areas was restricted due to the small size of the wound. This led to 2 to 5 measurement areas per burn wound. The validity was obtained by correlating the LDI color code of each measurement area to the associated $\Delta T$ of this measurement area. Thus, we assessed the ability of $\Delta T$ to distinguish between different burn wound HPs. For the validity analysis, the $\Delta T$ value of the first thermography measurement was used. 


\subsection{Statistical Analysis}

Data were analyzed using SPSS, Version 21.0 (IBM Corp., Armonk, New York). General patient characteristics were documented. The interobserver reliability was expressed by the intraclass correlation coefficient $\left.\left(\mathrm{ICC}_{\text {inter }}\right)\right)^{21}$ The $\mathrm{ICC}_{\text {inter }}$ was calculated using three variance components, obtained by a random-effects model [analysis of variance (ANOVA)]. ${ }^{15,21}$ Variance is the statistical term that is used to indicate variability.

- Patient variance $\left(\sigma_{\mathrm{pat}}^{2}\right)$ : variance due to systematic differences between "true" scores of patients.

- Observer variance $\left(\sigma_{\text {obs }}^{2}\right)$ : variance due to systematic differences between observers.

- Random error variance $\left(\sigma_{\text {error }}^{2}\right)$ : residual variance, partly due to the unique combination of patients and observers, and in addition to some random error.

The $\mathrm{ICC}_{\text {inter }}$ is the ratio between the patient variance and total variance: ICC $=\sigma_{\text {pat }}^{2} /\left(\sigma_{\text {pat }}^{2}+\sigma_{\text {obs }}^{2}+\sigma_{\text {error }}^{2}\right)$. An ICC value of 0.7 was considered as a minimum requirement for acceptable results. ${ }^{15}$

Furthermore, two parameters of the measurement error were calculated; the standard error of measurement (SEM) and the limits of agreement (LoA). These parameters are expressed on the actual scale of measurement. The SEM was obtained using the equation: $\mathrm{SEM}=\sqrt{ }\left(\sigma_{\mathrm{obs}}^{2}+\sigma_{\text {error }}^{2}\right)$. This leads to LoA of: mean difference $\pm 1.96 \times \mathrm{SEM} \times \sqrt{ } 2 .^{15,21}$ By definition, $95 \%$ of the differences between two measurements lie between these LoA. The LoA were indicated in a Bland and Altman plot, representing the absolute agreement between two temperature measurements. ${ }^{22}$ In this plot, the mean $\Delta T$ of the two measurements was plotted on the $x$-axis, against the difference between the $\Delta T$ values on the $y$-axis. ${ }^{22}$

To assess the validity, we compared the LDI color categories (ordinal scale) to the $\Delta T$ values (continuous scale) by ANOVA. We used receiver operating characteristic (ROC) curves to determine the ability of thermography to discriminate between burn wound HPs. In these curves, the true positive rate (sensitivity) is plotted against the false positive rate (1-specificity). The area under the ROC curve can be calculated and is a measure of how well $\Delta T$ can discriminate between the burn wound HPs expressed by LDI. The area under the ROC curve has a maximum value of 1.0 ; a value of 0.5 , represented by the diagonal, means that the measurement instrument under study (i.e., thermography) cannot distinguish between burn wound HPs. ${ }^{15}$ Finally, the distribution of burn wounds on $\Delta T$ was expressed using a visual method and one optimal $\Delta T$ cutoff value was determined with maximum sensitivity and specificity. ${ }^{23} \mathrm{We}$ did this for the distinction between burn wounds that heal spontaneously ( $\mathrm{HP}<14$ days and HP 14 to 21 days combined) and burn wounds that require surgical treatment (HP $>21$ days).

\section{Results}

\subsection{Patient and Burn Wound Characteristics}

Fifty burn wounds of 35 patients (Caucasians) were measured. Patient and burn wound characteristics are presented in Table 1. Median burn wound size was $2 \%$ TBSA, ranging from $0.5 \%$ to $12 \%$. At the time of assessment, burn wounds were managed using three different topical ointments: 35 (70\%) of wounds
Table 1 Patient and burn wound characteristics.

\begin{tabular}{lcr} 
& Value, $N$ & $\%$ \\
\hline Burn wounds & 50 & \\
Patients & 35 & \\
Sex & & $57 \%$ \\
Male & 20 & $43 \%$ \\
Female & 15 &
\end{tabular}

Age of patient, years

Median (range)

45 (18 to 81$)$

Assessment, postburn day

Median (range)

Cause of burn wound

$\begin{array}{lcc}\text { Flame } & 17 & 34 \% \\ \text { Scald } & 24 & 48 \% \\ \text { Contact } & 5 & 10 \% \\ \text { Chemical } & 4 & 8 \%\end{array}$

Burn wound location

\begin{tabular}{lll} 
Trunk & 11 & $22 \%$ \\
Arms & 20 & $40 \%$ \\
Legs & 19 & $38 \%$ \\
\hline
\end{tabular}

were treated by Flamazine, $14(28 \%)$ by Flaminal $^{\circledR}$, and 1 (2\%) by Fucidin ${ }^{\circledR}$.

\subsection{Reliability}

All 50 burn wounds were included for the reliability analysis. The variance components were assessed at 5.09 (patients), 0.00 (observers), and 0.05 (error). By means of these components, the $\mathrm{ICC}_{\text {inter }}$ was found to be 0.99 , expressing the correlation between the $\Delta T$ scores of two measurements. Subsequently, the SEM was calculated at $0.22^{\circ} \mathrm{C}[\sqrt{ }(0.00+0.05)]$. In addition, the lower LoA was assessed at $-0.58^{\circ} \mathrm{C}$ and the upper LoA at $0.64^{\circ} \mathrm{C}$, in view of the fact that the mean difference was $0.03^{\circ} \mathrm{C}$. The LoA were plotted to indicate the absolute agreement between two measurements (Fig. 4).

\subsection{Validity}

To assess the validity of thermography, we assigned 179 measurement areas in the same 50 burn wounds according to the standardized algorithm. The distribution of measurement areas and the mean $\Delta T$ value of all measurement areas within each burn wound category are given in Table 2 .

Two ROC curves were obtained to determine how well $\Delta T$ can differentiate between the three LDI categories and thus between the different burn wound outcomes. The estimated mean $( \pm \mathrm{SE})$ area under the ROC curve for thermography 


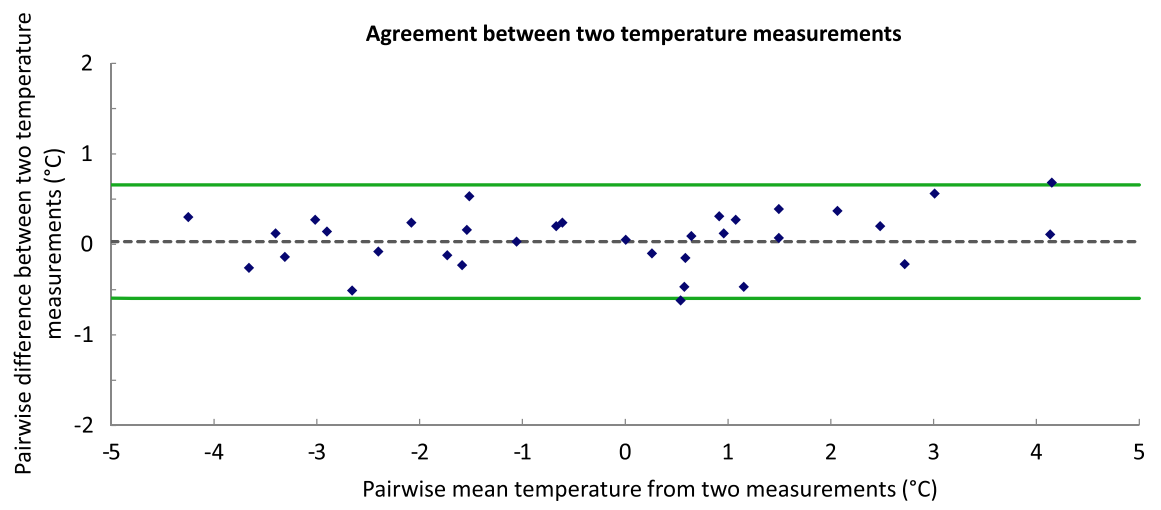

Fig. 4 Bland and Altman plot with the LoA (continuous lines), indicating the absolute agreement between two measurements. Note that the mean difference (dotted line) is nearly zero, indicating that there is no systematic difference between the two measurements.

Table 2 Number of measurement areas and mean $\Delta T$ values for each burn wound category, assessed by means of LDI.

\begin{tabular}{|c|c|c|c|c|}
\hline & $\mathrm{HP}<14$ days & HP 14 to 21 days & $\mathrm{HP}>21$ days & $p$-value \\
\hline Measurement areas, $N(\%)$ & 77 (43\%) & $39(22 \%)$ & $63(35 \%)$ & \\
\hline Mean $\Delta T,{ }^{\circ} \mathrm{C}(95 \% \mathrm{Cl})$ & 1.97 (1.59 to 2.36$)$ & $0.14(-0.22$ to 0.50$)$ & $-1.40(-1.78$ to -1.03$)$ & $<0.001^{\mathrm{a}}$ \\
\hline
\end{tabular}

Note: HP, healing potential.

${ }^{\mathrm{a} A N O V A .}$

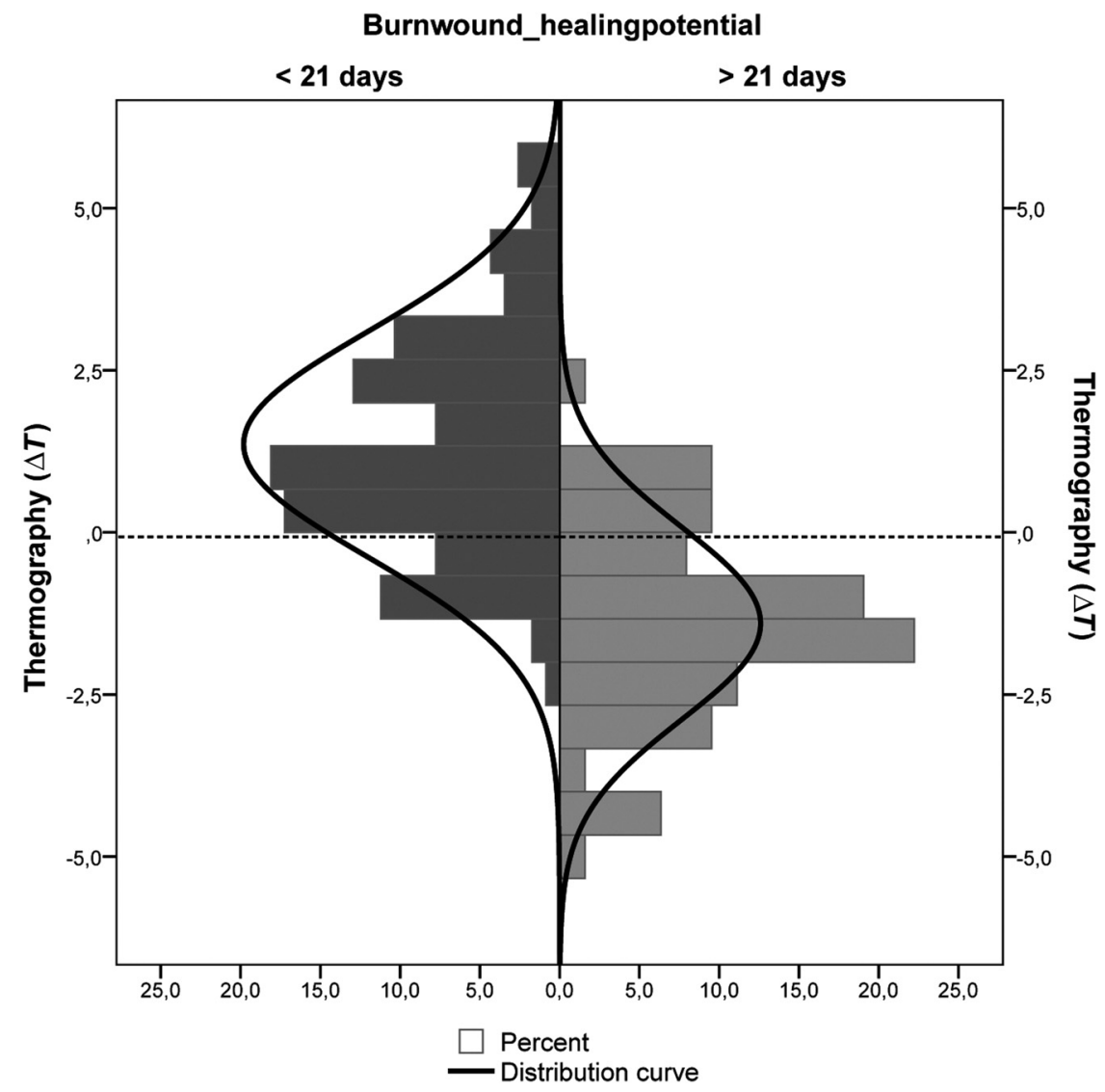

Fig. 5 Visual method expressing the distribution on $\Delta T$ of all burn wounds with HP $\leq 21$ days (HP $<14$ and HP 14 to 21 combined) versus HP $>21$ days. The dotted line shows the optimal cutoff point of $-0.07^{\circ} \mathrm{C}$ according to the ROC analysis based on the normal distribution curves. 
was $0.82 \pm 0.04(95 \%$ CI 0.74 to 0.89$)$ for the discrimination between burn wound HP $<14$ days and HP 14 to 21 days, and $0.80 \pm 0.04$ (95\% CI 0.72 to 0.89 ) for the discrimination between burn wound HP 14 to 21 days and HP >21 days. One $\Delta T$ cutoff value with maximum sensitivity and specificity was obtained that differentiates between all burn wounds that will heal spontaneously (HP $<14$ days and HP 14 to 21 days) and burn wounds that require surgical treatment (HP $>21$ days). The optimal cutoff point was $-0.07^{\circ} \mathrm{C}$ (sensitivity $80 \%$; specificity $80 \%$ ), as illustrated in Fig. 5.

\section{Discussion}

The objective of this study was to assess the reliability and validity of thermography for measuring burn wound HP. The $\mathrm{ICC}_{\text {inter }}$ of 0.99 corresponds to a very high correlation between two temperature measurements, indicating an excellent reliability. However, the ICC is only a measure of correlation but it does not provide any information on the measurement error. ${ }^{21}$ Therefore, two parameters of the measurement error were obtained: the absolute agreement between two measurements, expressed by the LoA, and the SEM. An important advantage of these parameters is that they are expressed on the actual scale of measurement $\left({ }^{\circ} \mathrm{C}\right)$, which promotes clinical interpretation. ${ }^{21}$ The SEM was calculated at $0.22^{\circ} \mathrm{C}$, which reflects the standard deviation around a single measurement. The LoA are based on this $\mathrm{SEM}$ value: $\mathrm{LoA}=$ mean difference $\pm 1.96 \times \mathrm{SEM} \times \sqrt{ } 2$. To guarantee that a $\Delta T$ change is unlikely to be due to the measurement error, a significance level of 0.05 is used, which corresponds to 1.96 . The $\mathrm{LoA}$ of $-0.58^{\circ} \mathrm{C}$ and $0.64^{\circ} \mathrm{C}$ show an acceptable variation in two $\Delta T$ measurements. To our knowledge, these are important findings since the reliability and agreement parameters of thermography have not been defined in prior research on burn wounds. When obtaining serial thermal measurements on consecutive postburn days, for example, it is of great importance that the instrument is able to perform repeated measurements that are free from measurement error. ${ }^{21}$ Moreover, by determining the agreement between two measurements, one can decide whether or not the values of different observers can be used interchangeably. ${ }^{21}$ Two factors may have contributed to the good reliability results. First, the highly sensitive thermal camera, the Xenics Gobi-384, which allows detection of temperature differences as small as $0.05^{\circ} \mathrm{C}$. Second, a short time interval between two measurements was chosen to ascertain that a stable population was assessed.

Next to reliability, we performed a validity analysis of thermography. Although this can be a difficult process (e.g., because of the required sample size or for the reason that it is challenging to select an accurate reference standard), we emphasize that it is of great importance to assess this clinimetric feature before the implementation of a measurement tool in clinical practice is considered. A recent study only examined the accuracy of thermography by calculating the correlation coefficient. ${ }^{24}$ Moreover, their accuracy was based on a study population of 20 patients. As a result, the two most important subgroups (i.e., burn wounds that healed in 14 to 21 days and burn wounds that took $>21$ days to heal) consisted of only 2 and 5 patients, respectively. In the current study, the validity of thermography was assessed using ROC curves. These curves express how well a $\Delta T$ value can distinguish between different burn wound HPs. Both areas under the ROC curve of 0.82 and 0.80 express a good discriminative value of $\Delta T$ for measuring burn wound HP. Subsequently, a $\Delta T$ cutoff value with corresponding maximum sensitivity and specificity was determined. This value is important for the use of thermography in clinical practice and has previously only been determined in an animal experiment or in a small number of (pediatric) burn patients. ${ }^{13,14}$ We obtained an optimal $\Delta T$ cutoff value of $-0.07^{\circ} \mathrm{C}$, differentiating between all burn wounds that are expected to heal and can primarily be treated conservatively (HP $<14$ days and HP 14 to 21 days), and burn wounds that require surgical treatment ( $\mathrm{HP}>21$ days). If the burn wound HP is $>21$ days, one can decide to accelerate the intervention (i.e., excision and skin grafting). These results are in line with previous research by Singer et al. ${ }^{25}$ who found a cutoff value of $0.1^{\circ} \mathrm{C}$, which was rounded to $0^{\circ} \mathrm{C}$ for simplification. The $80 \%$ sensitivity and $80 \%$ specificity associated with our cutoff value are good, but we think that these values can be improved. We encountered a few drawbacks in this study that may explain the validity results. Images obtained by LDI did not correspond to 1:1 with the thermography images, as the thermography camera was sometimes positioned at a slightly different angle or distance. This made it more difficult to correlate the exact same measurement areas, even though we applied the standardized algorithm on both thermography and LDI images. Especially within heterogeneous burn wounds, this may have impaired the results. Furthermore, a relatively high number of $\Delta T$ values observed in the distal extremities (hands and feet) tend to differ from what is expected based on the LDI results. Our hypothesis is that the temperature variation in distal extremities results in a $\Delta T$ which is influenced by the anatomical location rather than the burn wound. ${ }^{26}$ Unfortunately, the amount of burns on distal extremities was too small in our study to perform an acceptable subgroup analysis (8/50 burn wounds). In these eight patients, no clear tendency was found. Third, standard subjective burn wound assessment by our burn clinicians was not taken into account in this study. As with the use of LDI in daily practice, we think that the combination of thermography with subjective assessment (i.e., an add-on test) will result in even better validity. ${ }^{8,27}$ Furthermore, new handheld thermography cameras (including smart phone application) became available over the last months that are able to capture a thermal reading and standard picture at the same time. ${ }^{28}$ The system subsequently blends both images, providing evaluation of the exact burn wound area of interest. It would be interesting to examine these new cameras and to conduct a prospective study determining burn wound HP using the given $\Delta T$ cutoff value.

\section{Conclusion}

In this paper, the first clinimetric evaluation of thermography for measuring burn wound HP was performed. We conclude that thermography has a good reliability, as indicated by the high $\mathrm{ICC}_{\text {inter }}$ of 0.99 and the fact that there was no systematic difference between two measurements. Moreover, we obtained an optimal $\Delta T$ cutoff value of $-0.07^{\circ} \mathrm{C}$ associated with $80 \%$ sensitivity and $80 \%$ specificity, which leads to a good validity in the assessment of burn wounds. These are important findings in the search for measurement tools that can improve treatment decisions and therefore the outcome of burns. In addition, thermography is an affordable and very suitable technique, allowing easy and fast measurements. Our findings encourage further research into thermography and emphasize that this technique may become a gold standard in the clinical assessment of burn wounds in the future. 


\section{Acknowledgments}

The Xenics thermography camera was supplied free of charge for the duration of this study. This research was supported by the Dutch Burns Foundation (Grant No. 13.107). None of the authors has a financial interest in any of the products, devices, or drugs mentioned in this paper.

\section{References}

1. H. Goei et al., "Long term scar quality in burns with three distinct healing potentials: a multicenter prospective cohort study," Wound Repair Regen. 24, 721-730 (2016).

2. M. B. van der Wal et al., "Outcome after burns: an observational study on burn scar maturation and predictors for severe scarring," Wound Repair Regen. 20(5), 676-687 (2012).

3. J. C. Jeng et al., "Laser Doppler imaging determines need for excision and grafting in advance of clinical judgment: a prospective blinded trial," Burns 29(7), 665-670 (2003).

4. S. Monstrey et al., "Assessment of burn depth and burn wound healing potential," Burns 34(6), 761-769 (2008).

5. D. M. Heimbach et al., "Burn depth estimation-man or machine," J. Trauma 24(5), 373-378 (1984).

6. Z. B. Niazi et al., "New laser Doppler scanner, a valuable adjunct in burn depth assessment," Burns 19(6), 485-489 (1993).

7. A. D. Jaskille et al., "Critical review of burn depth assessment techniques: part II. Review of laser Doppler technology," J. Burn Care Res. 31(1), 151-157 (2010).

8. H. Hoeksema et al., "Accuracy of early burn depth assessment by laser Doppler imaging on different days post burn," Burns 35(1), 36-45 (2009).

9. S. A. Pape, C. A. Skouras, and P. O. Byrne, "An audit of the use of laser Doppler imaging (LDI) in the assessment of burns of intermediate depth," Burns 27(3), 233-239 (2001).

10. R. Mladick, N. Georgiade, and F. Thorne, "A clinical evaluation of the use of thermography in determining degree of burn injury," Plast. Reconstr. Surg. 38(6), 512-518 (1966).

11. M. E. Hackett, "The use of thermography in the assessment of depth of burn and blood supply of flaps, with preliminary reports on its use in Dupuytren's contracture and treatment of varicose ulcers," Br. J. Plast. Surg. 27(4), 311-317 (1974).

12. J. Hardwicke et al., "A pilot evaluation study of high resolution digital thermal imaging in the assessment of burn depth," Burns 39(1), 76-81 (2013).

13. J. D. Medina-Preciado et al., "Noninvasive determination of burn depth in children by digital infrared thermal imaging," J. Biomed. Opt. 18(6), 061204 (2013).
14. A. Renkielska et al., "Static thermography revisited-an adjunct method for determining the depth of the burn injury," Burns 31(6), 768-775 (2005).

15. H. C. W. De Vet et al., Measurement in Medicine. A Practical Guide, Cambridge University Press, Cambridge, United Kingdom (2011).

16. A. R. Feinstein, "An additional basic science for clinical medicine: IV. The development of clinimetrics," Ann. Intern. Med. 99(6), 843-848 (1983).

17. S. A. Pape et al., "Burn wound healing time assessed by laser Doppler imaging (LDI). Part 1: derivation of a dedicated colour code for image interpretation," Burns 38(2), 187-194 (2012).

18. S. M. Monstrey et al., "Burn wound healing time assessed by laser Doppler imaging. Part 2: validation of a dedicated colour code for image interpretation," Burns 38(2), 195-202 (2012).

19. R. P. Cole et al., "Thermographic assessment of burns using a nonpermeable membrane as wound covering," Burns 17(2), 117-122 (1991).

20. P. D. Verhaegen et al., "Sustainable effect of skin stretching for burn scar excision: long-term results of a multicenter randomized controlled trial," Burns 37(7), 1222-1228 (2011).

21. H. C. de Vet et al., "When to use agreement versus reliability measures," J. Clin. Epidemiol. 59(10), 1033-1039 (2006).

22. J. M. Bland and D. G. Altman, "Statistical methods for assessing agreement between two methods of clinical measurement," Lancet 327(8476), 307-310 (1986).

23. H. C. de Vet et al., "Minimally important change determined by a visual method integrating an anchor-based and a distribution-based approach," Qual. Life Res. 16(1), 131-142 (2007).

24. A. Burke-Smith, J. Collier, and I. Jones, "A comparison of non-invasive imaging modalities: infrared thermography, spectrophotometric intracutaneous analysis and laser Doppler imaging for the assessment of adult burns," Burns 41(8), 1695-1707 (2015).

25. A. J. Singer et al., "Infrared thermal imaging has the potential to reduce unnecessary surgery and delays to necessary surgery in burn patients," J. Burn Care Res. (2015).

26. W. P. Zhu and X. R. Xin, "Study on the distribution pattern of skin temperature in normal Chinese and detection of the depth of early burn wound by infrared thermography," Ann. N. Y. Acad. Sci. 888, 300313 (1999).

27. A. Van den Bruel et al., "The evaluation of diagnostic tests: evidence on technical and diagnostic accuracy, impact on patient outcome and costeffectiveness is needed," J. Clin. Epidemiol. 60(11), 1116-1122 (2007).

28. J. T. Hardwicke, O. Osmani, and J. M. Skillman, "Detection of perforators using smartphone thermal imaging," Plast. Reconstr. Surg. 137(1), 39-41 (2016).

Biographies for the authors are not available. 